\title{
Assistência de enfermagem prestada ao paciente estomizado no período perioperatório
}

\author{
Nursing care provided to ostomized patients in the perioperative period
}

\section{Atención de enfermería brindada a pacientes ostomizados en el período perioperatorio}

Wendy Larissa Costa da Silva ${ }^{1 *}$, Maíra Gonçalves de Melo ${ }^{1}$, Neuza Alves Cardoso ${ }^{1}$, Ana Cléo Borges Maia ${ }^{1}$, Antonio Carlos dos Santos Firmiano ${ }^{1}$, Larissa dos Santos Matos ${ }^{1}$, Aline Priscila Rodrigues Mineiro ${ }^{1}$, Kleyton dos Santos Tavares ${ }^{1}$, Safira Rayme Albuquerque Costa ${ }^{2}$, Alexia Eduarda Pantoja da Costa ${ }^{3}$.

\section{RESUMO}

Objetivo: Identificar em literaturas científicas as intervenções de enfermagem prestadas ao paciente estomizado no período perioperatório. Métodos: Trata-se de uma revisão de literatura, utilizando artigos originais e de revisão, em português, publicados entre 2015 a 2020, obtidos através de coletas nas seguintes bases de dados Scientific Electronic Library Online (SCIELO), Literatura Latino-Americana e do Caribe em Ciências da Saúde (LILACS) e ACERVO+ no período de janeiro a junho de 2020. Resultados: Observou-se que os pacientes estomizados necessitam de informações a respeito dos cuidados físicos, psicológicos e socias. Sendo assim, o enfermeiro entra como ferramenta essencial no planejamento da assistência desses pacientes, que inclui os cuidados desde o diagnóstico (fase pré-operatória) até a preparação para a alta hospitalar (fase pós-operatória). Considerações finais: Então se faz necessária à elaboração de novas pesquisas que abordem a efetividade desses cuidados prestados ao paciente estomizado, visando o aprimorando do conhecimento que influencia diretamente em uma prática adequada no atendimento pré, trans e pós-operatório.

Palavras-chave: Ostomia, Assistência de enfermagem, Autocuidado, Complicações.

\begin{abstract}
Objective: To identify the nursing interventions provided to ostomized patients in the perioperative period in scientific literature. Methods: This is a literature review, using original and review articles, in Portuguese, published between 2015 and 2020, obtained through collections in the following databases Scientific Electronic Library Online (SCIELO), Latin American Literature and Caribbean in Health Sciences (LILACS) and ACERVO+ from January to June 2020. Results: It was observed that ostomized patients need information regarding physical, psychological and social care. Thus, the nurse enters as an essential tool in the planning of care for these patients, which includes care from diagnosis (preoperative phase) to preparation for hospital discharge (postoperative phase). Final considerations: So it is necessary to develop new research that addresses the effectiveness of this care provided to ostomized patients, aiming at improving knowledge that directly influences an appropriate practice in pre, trans and postoperative care.
\end{abstract}

Keywords: Ostomy, Nursing care, Self-care, Complications.

1 Escola Superior Madre Celeste (ESMAC), Ananindeua - PA. *E-mail: wendylarissas@gmail.com

2 Escola Superior da Amazônia (ESAMAZ), Belém - PA.

3 Universidade da Amazônia (UNAMA), Ananindeua - PA. 


\section{RESUMEN}

Objetivo: Identificar las intervenciones de enfermería que se brindan a los pacientes ostomizados en el período perioperatorio en la literatura científica. Métodos: Se trata de una revisión de la literatura, utilizando artículos originales y de revisión, en portugués, publicados entre 2015 y 2020, obtenidos a través de colecciones en las siguientes bases de datos Scientific Electronic Library Online (SCIELO), Latin American and Caribbean in Health Sciences (LILACS) y ACERVO+ a partir de enero a junio de 2020. Resultados: Se observó que los pacientes ostomizados necesitan información sobre atención física, psicológica y social. Así, la enfermera entra como herramienta fundamental en la planificación de la atención de estos pacientes, que incluye la atención desde el diagnóstico (fase preoperatoria) hasta la preparación para el alta hospitalaria (fase postoperatoria). Consideraciones finales: Por lo que es necesario desarrollar nuevas investigaciones que aborden la efectividad de esta atención brindada a los pacientes ostomizados, con el objetivo de mejorar los conocimientos que inciden directamente en una práctica adecuada en los cuidados pre, trans y postoperatorios.

Palabras clave: Ostomía, Cuidado de enfermera, Autocuidado, Complicaciones.

\section{INTRODUÇÃO}

A palavra estomia é de origem grega a partir do étimo stoma, que corresponde à ideia de "boca". No momento atual, considerando a grafia brasileira e por consenso entre especialistas, adota-se a nomenclatura estomia/estoma e estomizado, porém o termo ostomia/ostomizado ainda é usado quando da referência a nomes vinculados a publicações governamentais. A estomia tem sua origem em 305 A. C., cuja mesma é citada em uma passagem bíblica, onde Praxógoras de Kos teria realizado uma cirurgia que apresentava um ferimento abdominal (MONTEIRO AKC, et al., 2020).

A causa mais comum de ocorrências de colostomia é o câncer colorretal ou doença diverticular, a estimativa do ano de 2014 de casos novos de câncer de reto e cólon no Brasil foi de 15.070 casos na população masculina e 17.530 na feminina, o que equivale a um risco estimado de 15,44 casos novos em relação a 100 mil homens e 17,24 a cada 100 mil mulheres. Resslatando que se a doença for diagnosticada em estágio inicial há chances de um bom prognóstico (OLIVEIRA IV, et al., 2018).

As cirurgias gastrointestinais são indicadas quando o sistema não está funcionando normalmente, por motivo patológico, trauma ou por má formação congênita no qual o ser humano não consegue eliminar de forma fisiológica tende-se a necessidade de realizar os estomas. A estomia é uma exteriorização da qual a nomeação diferenciasse de acordo com o segmento corporal em que será realizada a cirurgia, podendo ser classificada em: estomias respiratórias, alimentares, intestinais, urinárias e outras (SILVA NM, et al., 2017).

As estomias alimentares são realizadas com o objetivo de administrar alimentos pastosos e/ou liquídos. Dependendo do local que são realizados os procedimentos cirúrgicos podem ser classificados como: jejunostomia quando é designado o acesso ao jejuno através da parade abdominal proximal e gastrostomia que ocorre diretamente ao estômago. Tratando-se de estomias intestinais são definidas em em ileostomia e colostomia, respectivamente, como intervenções cirúrgicas feitas pela abertura de segmento ileal e cólico na parede abdominal, com objetivo de eliminar o conteúdo fecal (FONSECA AZ, et al., 2017).

A classificação da colostomia pode ser: colostomia ascendente, transversa, descendente e sigmoide. Nesse período, as fezes irão se apresentar de várias formas: liquidas, semi-líquidas, pastosas e sólidas, até a readaptação do sistema com a nova estrutura. Temos também, a colostomia úmida em alça, que no mesmo estoma ocorre a saída de fezes e urina. A ileostomia que é o acesso ao estômago através da parte final do intestino delgado, ocorre quando por algum motivo há a dificuldade da passagem das fezes pelo intestino grosso, logo o conteúdo fecal é mais liquído do que o eliminado por uma colostomia (ESPIRÍTO SANTO, 2017). 
A estomia urinária é chamada de urostomia, realizada através de intervenção cirúrgica, é feito um estoma para dispersão de urina e, esse procedimento envolve todo sistema urinário. São classificadas de acordo com o local que ocorre o procedimento. Nefrostostomia ou pielostomia se derivadas diretamente dos rins, ureterostomia quando há a exteriorização de um ureter, cistostomia/vesicostomia se advinda da bexiga (FONSECA AZ, et al., 2017).

A estomia não é um procedimento isento de complicações, mesmo utilizando as técnicas cirúrgicas corretas. Algumas das complicações mais recorrentes são: abcesso, dermatite, edema, estenose, foliculite, varizes periestomais, hemorragia, hérnia, necrose, prolapso e retração (SILVA NM, et al., 2017).

Dependendo do quadro clínico apresentado pelo paciente o estoma será provisório ou permanente, atingindo o paciente de forma emocional e física, tendo aspectos positivos ou negativos a resultar de sua adaptação a essa nova circunstância. Estomas permanentes são aqueles que a anatomia alterada proíbe 0 restabelecimento da continuidade gastrintestinal normal e permanece no paciente até findar a sua vida, já a provisória é aquela em que, no procedimento cirúrgico inicial, foram mantidas as estruturas que possibilitam o funcionamento fisiológico do trato gastrointestinal, assim sendo possível retirar em até 12 meses (MONTEIRO AKC, et al., 2020).

Até a década de 50 , os pacientes que eram operados por conta do câncer de cólon, evacuavam pelo abdômen, porém quando a irmã da enfermeira dinamarquesa Elisa sofreu com esta doença ela teve a magnificente ideia de melhorar a qualidade de vida da mesma, criando assim a bolsa de colostomia. As bolsas para estomia são disponibilizados pelo Sistema Único de Saúde (SUS), visando à prevenção de complicações, adjuvantes dermatológicos são distribuídos desde que o paciente seja cadastrado, por tanto a importância de notificação para liberação de recursos e garantia de cuidado (LIRA JAC, et al., 2016; BORGES EL, 2016).

Em 1930, no American Journal of Nursing, pela primeira vez foram descritos cuidados referentes a pessoas ostomizada, onde o autor ressaltava que o estoma é considerado uma deficiência de caráter coletivo e individual. A categoria de estomaterapia, foi instituída em 1958, em referência aos profissionais especializados no cuidado desses pacientes, sendo reconhecida posteriormente como uma especialidade da enfermagem (NOGUEIRA LT, 2019).

$\mathrm{Na}$ realização da estomia, o estomaterapeuta trabalha junto ao cirurgião e é responsável por fazer a demarcação local para a cirurgia e acompanhamento de todo processo de recuperação pós-cirurgia, onde as consultas de enfermagem se fazem indispensáveis e corroboram para segurança do procedimento e qualidade no atendimento (MELO MC, et al., 2020).

Os pacientes que são submetidos a estomia, passam por diversas mudanças na sua rotina, desde a forma como se vestir, hábitos alimentares á problemas psicossocias decorrentes do processo adaptativo da nova condição de saúde. As questões relacionadas ao isolamento social, distúrbios do sono e até mesmo preocupações ligadas ao financeiro são sentimentos vivenciados diariamente pelos mesmos e que necessitam de atenção especial (ESPIRÍTO SANTO, 2017).

Neste contexto, para o planejamento da assistência a esses pacientes é inserido os cuidados da equipe de enfermagem, que os assisti desde o diagnóstico até a preparação para a alta hospitalar. Alguns estudos afirmam que o conhecimento do paciente a cerca da sua situação, os influencia diretamente no processo de adaptação, devido isso, é de extrema importância o suporte psicológico e a educação em saúde, com o objetivo de desenvolver a capacidade para o autocuidado (HEY AP e NASCIMENTO LA, 2017).

A assistência de enfermagem durante o período perioperatório deve ser traçada e organizada, principalmente em intervenções de educação em saúde que englobem orientações ao paciente e aos seus familiares a respeito do que precisam entender e compreender sobre os cuidados em domicílio que são primordiais no estado biopsicossocial do paciente (NEGRI EC, et al., 2019).

Diante da complexidade dos cuidados necessários que precisam ser realizados desde o pré-operatório ao pós operatório e do aumento de pacientes com estomias intestinais, decorrente do índice crescente de 
novos casos de câncer de cólon e reto no Brasil, a realização deste estudo visa a busca para identificar na literatura científica as principais intervenções da equipe de enfermagem voltadas ao período perioperatório. Dessa forma, o objetivo do estudo é identificar em literaturas científicas as intervenções de enfermagem prestadas ao paciente estomizado no período perioperatório.

\section{MÉTODOS}

Trata-de de uma revisão da literatura, de caráter exploratório. O estudo foi realizado através da busca online para o levantamento bibliográfico nas seguintes bases de dados Scientific Electronic Library Online (SCIELO), Literatura Latino-Americana e do Caribe em Ciências da Saúde (LILACS) e ACERVO+. Foi realizada utilizando-se os seguintes descritores "ostomia", "assistência de enfermagem", "autocuidado", "complicações". Foram incluídos artigos originais e de revisão, em língua portuguesa, publicados entre 2015 a 2020, que abordavam os cuidados prestados a pacientes estomizados no período perioperatório, os dados foram coletados em junho de 2020, obtendo 40 referências apenas pelos títulos, sendo avaliados posteriormente através dos critérios de exclusão, demonstrados através do fluxograma (Figura 1).

Figura 1 - Fluxograma dos critérios de exclusão e quatitativo dos estudos incluídos.

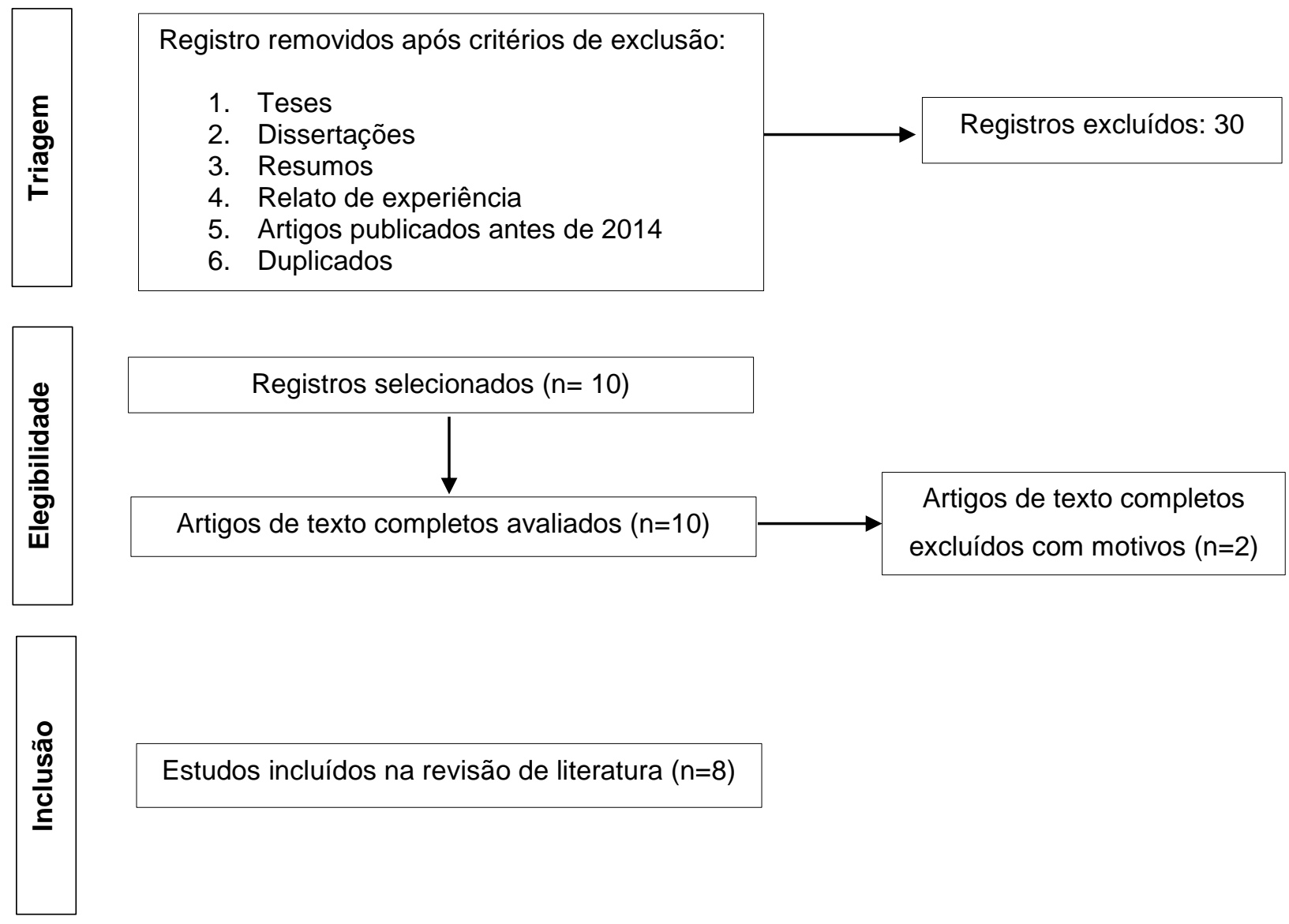

Fonte: Silva WLC, et al., 2020.

Para melhor entendimento da pesquisa, foi montado um quadro estruturado onde os artigos foram sumarizados com codificação, registro do título, autores, ano de publicação e base de dados. Não foi necessário submeter ao Comitê de Ética devido não envolver pesquisas diretas com seres humanos se tratando apenas de uma revisão de literatura, conforme constam na Resolução 466/2012 e 510/2016 do Ministério da Saúde. Dispõe de risco de plágio e visando beneficiar acadêmicos e profissionais da área de saúde, em especial a equipe de enfermagem, assim como a comunidade em geral. 


\section{RESULTADOS E DISCUSSÃO}

Dos 8 estudos analisados, três são revisões integrativas e cinco artigos originais e 7 foram publicados em revista de enfermagem e 1 em revista que aborda assuntos da saúde. No artigo A4 e A6, foram encontradas evidências dos cuidados de enfermagem no período pré-operatório, no A2, A7 e A8 cuidados relacionados ao período transoperatório e A1, A3 e A5 a respeito do período pós-operatório (Quadro 1).

Quadro 1 - Distribuição dos artigos selecionados.

\begin{tabular}{|c|c|c|c|c|}
\hline Código & Título & Autor/Ano & Base & Principais resultados \\
\hline $\mathrm{A} 1$ & $\begin{array}{c}\text { "Intervenções de enfermagem } \\
\text { para alta de paciente com } \\
\text { estomia intestinal: revisão } \\
\text { integrativa." }\end{array}$ & $\begin{array}{l}\text { AZEVEDO C, } \\
\text { et al. } 2015\end{array}$ & LILACS & $\begin{array}{l}\text { Avaliar aspectos do estoma; } \\
\text { controle dos efluentes do } \\
\text { estoma. }\end{array}$ \\
\hline $\mathrm{A} 2$ & $\begin{array}{c}\text { "Da formação à prática: A } \\
\text { percepção de supervisores de } \\
\text { enfermagem sobre os cuidados } \\
\text { em estomias." }\end{array}$ & $\begin{array}{l}\text { LIRA JAC, et } \\
\text { al. } 2016\end{array}$ & LILACS & $\begin{array}{c}\text { Planejamento de } \\
\text { enfermagem especifíco; } \\
\text { verificação e avaliação do } \\
\text { estoma de acordo com a } \\
\text { demarcação prévia. }\end{array}$ \\
\hline A3 & $\begin{array}{l}\text { "A visão dos enfermeiros } \\
\text { sobre as práticas } \\
\text { educativas direcionadas as } \\
\text { pessoas estomizadas." }\end{array}$ & $\begin{array}{l}\text { MAURICIO } \\
\text { VC, et al. } 2017\end{array}$ & SCIELO & $\begin{array}{c}\text { Informações claras e } \\
\text { objetivas que auxiliem no } \\
\text { autocuidado, principalmente } \\
\text { no momento da alta } \\
\text { hospitalar. }\end{array}$ \\
\hline A4 & $\begin{array}{l}\text { "Fatores de risco associados ao } \\
\text { desenvolvimento de } \\
\text { complicações do estoma de } \\
\text { eliminação e da pele } \\
\text { periestomal." }\end{array}$ & $\begin{array}{l}\text { PINTO IES, et } \\
\quad \text { al. } 2017\end{array}$ & SCIELO & $\begin{array}{c}\text { Informações a cerca do } \\
\text { diagnóstico; averiguação dos } \\
\text { antecedentes familiares; } \\
\text { anamnese geral. }\end{array}$ \\
\hline A5 & $\begin{array}{c}\text { "Autoimagem e autocuidado na } \\
\text { vivencia de pacientes } \\
\text { estomizados: o olhar da } \\
\text { Enfermagem." }\end{array}$ & $\begin{array}{l}\text { FREIRE DA, } \\
\text { et al. } 2017\end{array}$ & LILACS & $\begin{array}{c}\text { Incentivar o retorno a } \\
\text { atividades diárias, gerando o } \\
\text { sentimento de } \\
\text { independência,que reflete na } \\
\text { autoimagem e autocuidado. }\end{array}$ \\
\hline A6 & $\begin{array}{c}\text { "Cuidado e saúde em pacientes } \\
\text { estomizados." }\end{array}$ & $\begin{array}{l}\text { SILVA NM, et } \\
\text { al. } 2018\end{array}$ & LILACS & $\begin{array}{l}\text { Anamnese geral; } \\
\text { informações sobre rotina: } \\
\text { social e trabalho. }\end{array}$ \\
\hline A7 & $\begin{array}{l}\text { "Importância da atuação do } \\
\text { enfermeiro na demarcação do } \\
\text { estoma no pré-operatório } \\
\text { mediato." }\end{array}$ & $\begin{array}{l}\text { FREITAS } \\
\text { RCL, et al. } \\
2018\end{array}$ & ACERVO+ & $\begin{array}{l}\text { Demarcação prévia; } \\
\text { verificação e avaliação do } \\
\text { estoma de acordo com a } \\
\text { demarcação prévia; }\end{array}$ \\
\hline A8 & $\begin{array}{l}\text { "Qualidade de vida da pessoa } \\
\text { estomizada: relação com o } \\
\text { cuidado prestado na consulta } \\
\text { de enfermagem em } \\
\text { estomaterapia." }\end{array}$ & $\begin{array}{l}\text { MIRANDA } \\
\text { LSG, et al. } \\
\quad 2018\end{array}$ & SCIELO & $\begin{array}{c}\text { Estimulação da autonomia, } \\
\text { através de informações } \\
\text { repassadas a respeito da } \\
\text { bolsa coletora, higienização } \\
\text { e vestimentas. }\end{array}$ \\
\hline
\end{tabular}

Fonte: Silva WLC, et al., 2020.

De acordo com os artigos selecionados do Quadro 1, observou-se que os pacientes estomizados necessitam de informações a respeito dos cuidados físicos, psicológicos e socias. Sendo assim, o enfermeiro entra como ferramenta essencial no planejamento da assistência desses pacientes, que inclui os cuidados desde o diagnóstico (fase pré-operatória) até a preparação para a alta hospitalar (fase pós-operatória).

No artigo A4 e A6 foi possível perceber algumas intervenções da assistência de enfermagem na fase préoperatória como: o fornecimento de informações para o indivíduo acerca do diagnóstico e suas perspectivas,averiguação dos antecedentes familiares, antecedentes alérgicos, rotinas e problemas na eliminação intestinal; busca por informações sobre atividades diárias, sociais e de trabalho, além da demarcação prévia (MAURICIO VC, et al., 2017; SILVA NM, et al). 
No período pré-operatório o paciente apresenta ansiedade com relação ao procedimento cirúrgico, preocupações com sua família e trabalho, recuperação e condições físicas e psicológicas após a cirurgia. Especialmente, os pacientes candidatos a colostomia encontra-se preocupados com as mudanças no estilo de vida e alteração da imagem corporal, angustiam-se por não saber realizar os cuidados com a bolsa e nem como adquiri-la (SILVA CRDT, et al., 2017).

O profissional de enfermagem também deve assistir o paciente em relação ao choque emocional e psicológico ocasionado pela presença do estoma; deve repassar informações sobre o processo adaptativo relacionado ao uso da bolsa de ostomia; implementar a promoção da autoestima; incetivar a participação em grupos de apoio, institutos de caridade ou associações locais . O enfermeiro é o profissional que está em maior proximidade com o paciente, acredita-se que este seja o principal responsável em ensinar as habilidades manuais através de exercícios de destreza e agilidade para o autocuidado do paciente estomizado, o que preveni o isolamento social e a dependência de cuidado (BORGES EL, 2016; HEY AP e NASCIMENTO LA, 2017).

Detectou-se no artigo A2, A7 e A8 que no período trans-operatório o planejamento de enfermagem específico para este paciente envolve visita do enfermeiro do centro cirúrgico, padronização do equipamento a ser utilizado no pós-operatório imediato que facilite a avaliação do estoma (bolsa coletora de peça única, transparente e drenável), verificação e avaliação do estoma confeccionado em relação à demarcação prévia e do estado geral do paciente antes do encaminhamento ao serviço de recuperação pós-anestésica, além dos cuidados básicos desenvolvidos no centro cirúrgico pela equipe de enfermagem (PINTO IES, et al., 2017; MIRANDA LSG, et al., 2018).

Com a sistematização da assistência há melhora na dinâmica do atendimento, padronização de registros, adequação de informação ao paciente e baixo índice de complicação de pele periestoma, proporcionando ao paciente e família maior segurança, aptidão ao autocuidado e promovendo assim uma reabilitação social precoce (MOTA MS, et al., 2016).

As orientações de cuidados em relação à instalação das bolsas de estomia se referem às dificuldades de aderência e possíveis complicações, no qual o enfermeiro deve avaliar os riscos de funcionalidade e estimular o autocuidado, esclarecer quanto aos riscos de se instalar o equipamento próximo às proeminências ósseas, dobras cutâneas, cicatrizes e a importância da higienização adequada (FREIRE DA, et al., 2017).

O processo de enfermagem ocorre a partir do diagnóstico para prescrição de medidas que devem atender as necessidades do paciente a partir da consulta de enfermagem, com finalidade de reduzir ou minimizar os problemas potenciais advindos do estoma e com isso avaliar o impacto da estomia para o paciente e planejar as recomendações para o autocuidado (MIRANDA LSG, et al., 2018).

O enfermeiro percebendo as inseguranças a respeito da bolsa coletora deve repassar orientações de forma fácil e prática. Explicando sobre os equipamentos que estão disponíveis no mercado, além de ensinar na escolha da bolsa mais adequada para sua ostomia; discutindo e demonstrando como realizar a troca e esvaziamento da bolsa no domicílio; além de reinterar que o esvaziamento da bolsa deve ser feito quando estiver completado a metade de seu preenchimento, a clampear após o procedimento e orientando a respeito do uso do soro fisiológico ou água para o descolamento da bolsa (FREITAS RCL, et al., 2018).

O artigo A1, A5 e A3 expõe que no período pós-operatório é necessário observar e avaliar condições do estoma (localização, cor, tamanho e complicações); controlar o efluente do estoma de forma quantitativa e qualitativa; avaliar adaptação do sistema coletor; realizar a primeira troca do sistema coletor (48 a 72 horas após a cirurgia); ensinar ações específicas do autocuidado (troca e esvaziamento do dispositivo); retomar o ensino pré-operatório e planejar a alta hospitalar e estimular o retorno gradual às atividades da vida diária (AZEVEDO C, et al., 2015; LIRA JAC, 2016 et al; MAURICIO VC, et al., 2017).

Aa orientações fornecidas pelo enfermeiro sobre os cuidados com o estoma, são: informações em relação a aparência do estoma que deve ter aspecto úmido e brilhante, cor vermelho-cereja; orientar que depois de semanas após-cirurgia o estoma alterará sua aparência e dimensão, orientar quanto as complicações que podem ocorrer no pós-cirurgia, como: sinais de infecção, a inexistência de fezes durante ou mais que 24 horas 
e prolapso; orientar sobre os cuidados com o estoma durante o asseio, em relação a medição peródica do tamanho do estoma e como descartar todos os materiais utilizados durante o cuidado (OLIVEIRA IV, et al., 2018).

A maioria dos pacientes submetidos a cirurgias que ocasionam em ostomias intestinais não conhecem as mudanças que terão que conviver no pós-cirúrgico, como: hábitos alimentares saudavéis, vestimenta, alterações associadas a sexualidade como perda da libido e disfunção erétil. Logo é indispensável que o enfermeiro repasse orientações que irão os auxiliar no enfrentamento de tais mudanças, garantindo assim a continuidade do cuidado, a minimização das possíveis complicações e o aumento da qualidade de vida (MIRANDA LSG, et al., 2018).

Entende-se que o conhecimento do autocuidado permite ao paciente maior autonomia em relação a outras pessoas, pois ele mesmo realiza os cuidados necessários, através de técnicas básicas e adequadas, que possibilitam um cuidado seguro durante a realização do autocuidado, logo existe uma enorme preocupação em dos profissionais de enfermagem em preparar o paciente no pós-alta, visando facilitar a convivência com estoma nos cuidados em domicílio (OLIVEIRA IV, et al., 2018).

O fornecimento de orientações importantes muita das vezes é comprometido devido o encurtamento do tempo de internação, interferindo diretamente na eficácia do plano de alta hospitalar, e consequentemente no aumento de readmissões hospitalares decorrentes das complicações ocasionadas em domicílio (FREIRE DA, et al., 2017).

O enfermeiro como um dos profissionais responsáveis pelo paciente estomizado em relação ao ensino de práticas, deve combinar diversas formas de repassar orientações dos cuidados de forma clara e de fácil entendimento, através de informações escritas e demonstração de procedimentos básicos, criando assim um mecanismo de confiança entre profissional e paciente garantido que a assistência prestada a este seja completa e eficaz (ESPÍRITO SANTO, 2017).

Conforme os estudos avaliados, os cuidados relacionados a bolsa de ostomia apresentou maior número de intervenções, seguida pelas intervenções relacionadas ao cuidado da pele periestoma, logo isso insinua que intervenções relacionadas a área psicológica, ao apoio e orientações para o retorno da rotina diária não são eficientemente exploradas no mundo científico (NEGRI EC, et al., 2019).

Relacionado as necessidades psicológicas dos pacientes estomizados, um estudo realizado no Reino Unido com a finalidade de caracterizar fatores psicossocias relevantes para o cuidado com o estomizado, relatou que pacientes com ostomias, convivem desde sentimentos de ansiedade a preocupações sexuais, dessa maneira é indispensável que o profissional mantenha uma comunicação efetiva e consiga reconhecer estes sentimentos com o propósito de atenuar ou evitar o sofrimento psicológico (BRITO MAC, 2017).

Um estudo realizado no Brasil que objetivou entender os significados atribuídos a rotina dos pacientes estomizados e detalhar a cerca do conhecimento do estomizado sobre o autocuidado e identificar a relevância das orientações de enfermagem no processo pós-alta, possibilitou entender que as necessidades destes pacientes estavam, de forma geral, ligadas as mudanças que ocorrem no modo de vida, por conta da não aceitação estoma, e pela mudança da autoimagem, e que o profissional de enfermagem como educador assume papel primordial na ajuda no enfrentamento dessas dificuldades (MONTEIRO AKC, et al., 2020).

Os estudos evidenciam que não há muitos estudos realizados no Brasil, logo evidencia uma carência de materiais científicos nesta área. Mostrando a necessidade do desenvolvimentos de pesquisas que abordem cuidados específicos de pacientes estomizados no período perioperatório. Em relação aos peródicos cientifícos onde as pesquisas foram publicadas, com predominiação de periódicos de enfermagem. Alguns artigos especifícos da aéra de estomaterapia foram encontrados, porém não explanavam nenhum cuidados de enfermagem no período perioperatório. Ressalta-se que em todas as publicações os enfermeiros estavam como primeiro autor (SILVA CRDT, et al., 2017).

Devido a profissão de enfermargem ter a sua fundamentação voltada para o cuidado do paciente, é esperável que a maioria das publicações seja desta categoria profissional. E nesse contexto, é inserida a 
função do especialista estomaterapeuta, que é o profissional de saúde que possui capacitação para prestar cuidados ao estomizado, tanto da perspectiva técnica quanto psicológica, pois possui conhecimentos e competências especifícas, voltadas para a autonomia e promoção da autoestima, que certificam ao paciente estomizado uma melhor adaptação a sua nova condição de vida (SILVA NM, et al., 2017).

\section{CONSIDERAÇÕES FINAIS}

Através do estudo pudemos identificar a deficiência de materiais voltados a essa temática. Então se faz necessária à elaboração de novas pesquisas que abordem a efetividade desses cuidados prestados ao paciente estomizado, visando o aprimoramento do conhecimento que influencia diretamente em uma prática adequada no atendimento pré, trans e pós-operatório. Desta forma, possibilitando uma reabilitação precoce ao paciente estomizado durante estes processos, evitando ao máximo as complicações decorrentes dos cuidados inadequados. Estimulando os profissionais de enfermagem a terem um olhar holístico, compreendendo que o paciente necessita de orientações/cuidados físicos, psicológicos e sociais.

\section{REFERÊNCIAS}

1. AZEVEDO C, et al. Intervenções de enfermagem para alta de paciente com estomia intestinal: revisão integrativa. Rev Cubana Enferm, 2015;30(2).

2. BORGES EL. A atuação do enfermeiro na estomaterapia e a legislação brasileira: Avanços e crescimento da área. Recom. Minas Gerais. Maio, 2016; 6(2).

3. BRITO MAC. Fatores de risco associados ao desenvolvimento de complicações do estoma de eliminação e da pele periestomal. Rev. Enf. Ref, 2017; 4(15): 155-166.

4. ESPÍRITO SANTO. Secretaria de Saúde. Manual de orientação aos serviços de atenção às pessoas ostomizadas. Governo do Estado do Espírito Santo, 2017.

5. FONSECA AZ, et al. Fechamento de colostomia: fatores de risco para complicações. ABCD Arq Bras Cir Dig, 2017; 30(4): 231-234.

6. FREIRE DA, et al. Autoimagem e autocuidado na vivencia de pacientes estomizados: o olhar da Enfermagem. REME - Rev Min Enferm., 2017; 21(e-1019).

7. FREITAS RCL, et al. Importância da atuação do enfermeiro na demarcação do estoma no pré-operatório mediato. Revista Eletrônica Acervo Saúde (REAS), 2018. Vol. Sup. 11: S1567-S1573.

8. HEY AP, NASCIMENTO LA. A Pessoa com Estomia e o Fornecimento de Equipamentos Coletores e Adjuvantes pelo Sistema Único de Saúde. ESTIMA, 2017; 15(2): 92-99.

9. LIRA JAC, et al. Da formação à prática: A percepção de supervisores de enfermagem sobre os cuidados em estomias. Revista de enfermagem UERJ, 2016; 24(2): e14733.

10. MAURICIO VC, et al. A visão dos enfermeiros sobre as práticas educativas direcionadas as pessoas estomizadas. Esc. Anna Nery, 2017; 21(4): e20170003.

11. MELO MC, et al. Práticas de cuidado à criança estomizada: narrativas de familiares. Rev. Bras. Enferm, 2020; 73(2):18.

12. MIRANDA LSG, et al. Qualidade de vida da pessoa estomizada: relação com o cuidado prestado na consulta de enfermagem em estomaterapia. Esc. Anna Nery, 2018; 22(4): e20180075.

13. MOTA MS, et al. Repercussões no processo vivo de pessoas com estomas. Texto contexto - enferm, 2016; 25(1): e1260014.

14. MONTEIRO AKC, et al. Efecto de la intervención educativa en el postoperatorio de personas con estomias intestinales de eliminación: revisión sistemática. Enferm. glob. 2020; 19(57): 648-690.

15. NEGRI EC, et al. Construção e validação de cenário simulado para assistência de enfermagem a pacientes com colostomia. Texto contexto - enferm, 2019; 28: e20180199.

16. NOGUEIRA LT. Custos de equipamentos coletores e adjuvantes em pacientes com estomias de eliminação. REMERev Min Enferm, 2019; 23:e-116.

17. OLIVEIRA IV, et al. Cuidado e saúde em pacientes estomizados. Rev Bras Promoç Saúde, 2018; 31 (2): 1-9.

18. PINTO IES, et al. Fatores de risco associados ao desenvolvimento de complicações do estoma de eliminação e da pele periestomal. Rev. Enf. Ref, 2017; 5(15): 155-166.

19. SILVA NM, et al. Aspectos psicológicos de pacientes com estoma intestinal: revisão integrativa. Rev. LatinoAm. Enfermagem, 2017; 25: e2950.

20. SILVA CRDT, et al. Qualidade de vida de pessoas com estomias intestinais de eliminação. Acta paul. Enferm, 2017; 30(2): 144-151. 